\title{
Wolbachia strain wAlbB confers both fitness costs and benefit on Anopheles stephensi
}

\author{
Deepak Joshi ${ }^{1}$, Michael J McFadden ${ }^{1}$, David Bevins ${ }^{1}$, Fengrui Zhang ${ }^{1}$ and Zhiyong Xi, ${ }^{1}{ }^{*}$
}

\begin{abstract}
Background: Wolbachia is a maternally transmitted intracellular bacterium that is estimated to infect up to $65 \%$ of insect species, but it is not naturally present in Anopheles malaria vectors. Wolbachia-based strategies for malaria vector control can be developed either through population replacement to reduce vectorial capacity or through population suppression to reduce the mosquito population. We have previously generated An. stephensi mosquitoes carrying a stable wAlbB Wolbachia infection and have demonstrated their ability to invade wild-type laboratory populations and confer resistance to Plasmodium on these populations.
\end{abstract}

Methods: We assessed wAlbB-associated fitness by comparing the female fecundity, immature development and survivorship, body size, male mating competiveness, and adult longevity of the infected An. stephensi to that of wild-type mosquitoes.

Results: We found that WAlbB reduced female fecundity and caused a minor decrease in male mating competiveness. We also observed that wAlbB increased the life span of both male and female mosquitoes when they were maintained solely on sugar meals; however, there was no impact on the life span of blood-fed females. In addition, wAlbB did not influence either immature development and survivorship or adult body sizes.

Conclusions: These results provide significant support for developing Wolbachia-based strategies for malaria vector control.

Keywords: Wolbachia, Malaria, Anopheles, Fitness

\section{Background}

Wolbachia is a maternally transmitted intracellular bacterium that infects an estimated $65 \%$ of insect species [1,2] and $28 \%$ of mosquito species [3]. With such a broad host range, however, Wolbachia does not naturally infect the Anopheles malaria vectors or the primary dengue vector, Aedes aegypti. As a symbiotic bacterium of insects, Wolbachia can manipulate host reproduction in a number of "selfish" ways [2], resulting in its own spread into a population. Among these manipulations, cytoplasmic incompatibility $(\mathrm{CI})$ is the most common and is the only phenotype observed in mosquitoes [4]. CI results in early embryonic death when an uninfected female mates with an infected male. An infected female can produce infected, viable offspring when she mates

\footnotetext{
* Correspondence: xizy@msu.edu

${ }^{1}$ Department of Microbiology and Molecular Genetics, Michigan State University, East Lansing, MI 48824, USA

${ }^{2}$ Sun Yat-sen University - Michigan State University Joint Center of Vector Control for Tropical Diseases, Guangzhou, Guangdong 510080, China
}

with either an infected or an uninfected male, but an uninfected female can produce viable offspring only when she mates with an uninfected male. Therefore, CI provides a reproductive advantage to Wolbachia-infected females over uninfected females, resulting in spread of Wolbachia into populations [5-7].

As a reproductive parasite, Wolbachia is typically highly enriched in insect reproductive tissues such as ovaries and testes. Depending on the host and the length of the association, Wolbachia can also have a broad tissue distribution and be found in somatic tissues such as the midgut, fat body, and salivary gland [8]. This broad distribution has frequently been observed in a system involving recent Wolbachia/host association, and it has resulted in an alteration in the host's local physiological environment such that it has become resistant to pathogen infection $[7,9,10]$. Since its discovery, this Wolbachiamediated pathogen interference has been observed in both naturally and artificially infected insect hosts and has been 
demonstrated in a broad spectrum of microorganisms, including dengue virus, Chikungunya, Plasmodium, and filarial worms $[9,11-13]$.

The ability of Wolbachia to induce pathogen interference and also spread into mosquito vector populations makes it a potential biological agent for controlling both malaria and dengue $[5,6,9,10,14]$. The success of a recent field trial has indicated that Wolbachia can be deployed as a practical intervention strategy to control mosquito-borne diseases, with the potential for areawide implementation [6]. With the stable introduction of wAlbB Wolbachia into An. stephensi [7], we are now able to develop a strategy of this kind for malaria vector control. Toward this end, we have previously verified that $w \mathrm{AlbB}$ can confer some resistance to the human malaria parasite Plasmodium falciparum in the transinfected An. stephensi LB1 strain [7]. Seeding of naturally uninfected An. stephensi populations with infected females results in Wolbachia invasion of laboratory mosquito populations [7].

A mathematical model has predicted that the strength of the CI, maternal transmission efficacy, and Wolbachiaassociated fitness are three key parameters that determine the dynamics of Wolbachia in the population replacement process [15]. $w$ AlbB displays perfect maternal transmission and induces nearly complete CI in An. stephensi, but it also produces a reduced egg hatch rate in LB1 mosquitoes [7]. This reduction in egg hatch was not observed in the other two mosquito species carrying $w \mathrm{AlbB}$, the native host Ae. albopictus and the transinfected Ae. aegypti line [5,16]. In fact, Wolbachia was observed to produce a fitness benefit in these two species. In Ae. albopictus, Wolbachia (wAlbA and wAlbB)-infected females live longer, produce more eggs, and have higher hatch rates than do uninfected females [16]. An increase in life span was also observed in the transinfected Ae. aegypti females carrying $w \mathrm{AlbB}[10]$, and the transinfected Ae. albopictus males carrying $w$ Mel [17]; however, wMelPop reduced longevity in both native Drosophila species and transinfected Aedes mosquitoes [18,19].

The ability of $w$ AlbB to induce nearly complete CI when an LB1 male mates with a wild-type female supports the feasibility of using Wolbachia in a population suppression strategy. Derived from sterile insect technique (SIT), this strategy is also referred to as the incompatible insect technique (IIT) strategy, in which mass release of Wolbachia-infected males is used to induce CI matings with the wild-type females [20]. Success with this strategy has resulted in the eradication of Culex pipiens fatigans in a village in Burma and a recent effort to control the Polynesian tiger mosquito Ae. polynesiensis to eliminate lymphatic filariasis in the South Pacific $[21,22]$. In this strategy, the mating performance of LB1 males relative to the wild-type males is one of the key factors that facilitate the local suppression or eradication of An. stephensi.

Developing both population replacement and population suppression strategies to control An. stephensi requires a better understanding of Wolbachia-associated fitness in this mosquito species. In the present study, we compared the female fecundity, immature development and survivorship, body size, male mating competiveness, and adult longevity of infected LB1 and wild-type LIS mosquitoes. The results showed that wAlbB Wolbachia induces a fitness cost and also confers benefit on $A n$. stephensi, underscoring the complexity of WolbachiaAnopheles mosquito interactions.

\section{Methods}

\section{Ethics statement}

This study was carried out in strict accordance with the recommendations in the Guide for the Care and Use of Laboratory Animals of the National Institutes of Health. The protocols (03/14-036-00) were approved by the Michigan State University Institutional Animal Care and Use Committee.

\section{Mosquito lines}

The wild-type An. stephensi (Liston strain [LIS]) mosquitoes were provided by the Johns Hopkins Malaria Research Institute. The Wolbachia-infected An. stephensi LB1 strain used in these experiments was artificially generated via embryonic injection and back-crossed with uninfected wild-type males for at least four generations to reduce genetic bottlenecks [7]. The aposymbiotic line LBT was derived from LB1 as described previously [7]. The adult mosquitoes were maintained on sugar solution at $27^{\circ} \mathrm{C}$ and $85 \%$ humidity with a 12 -hr light/dark cycle according to standard rearing procedures. To initiate egg development, 5- to 7-day old adult females were fed on anesthetized BALB/C mice. Two days after bloodfeeding, oviposition sites (cups containing filter paper moistened with water) were placed inside cages to harvest the eggs. After two consecutive nights of egg collection, eggs were hatched, and larval trays were set up. The larval rearing conditions used in the assessment of the life history traits were the same ones used for the stock lines, with the density at 100 larvae $/ 660 \mathrm{ml}$ water in all larval rearing pans. All the treatments on mosquitoes in the experiments described below were run from three trays of pupae independently. In the mosquito colonies, approximately 1,200 adults with the sex ratio 1:1 (female : male) were maintained in a cage $(30 \times 30 \times 30$ inches $)$.

\section{Fecundity tests}

Females were randomly selected from the population cages containing 8-to 9-day-old adult mosquitoes and then transferred to new cages. The mosquitoes in these 
cages were fed on anesthetized BALB/c mice for $\sim 20 \mathrm{~min}$ or on commercial human or sheep blood, using a membrane feeding apparatus, for $\sim 30 \mathrm{~min}$. The unfed mosquitoes were then removed. Two days after blood-feeding, individual blood-fed females were transferred to a $50-\mathrm{ml}$ Falcon tube with a bottom lining of moist filter paper supported by water-soaked cotton. After two nights of egg collection, egg papers were immersed in hatching cups (50-ml plastic cups half filled with water). The edges of the hatching cups were kept moistened with an extra lining of filter paper in order to prevent egg death, which is most likely to happen when eggs come into contact with dry cup edges. The eggs were left there for hatching for 2 days. The following day, the egg hatch was scored under a dissecting microscope.

\section{Assessment of life history traits}

Approximately 100 larvae, hatched within $2 \mathrm{hr}$, were transferred to a plastic larval tray containing $660 \mathrm{ml}$ of distilled water. The larval diet was composed of powdered fish food (First Bites, Hikari Tropical) and cat food (Purina cat food chow, Nestlé Purina PetCare). The mixture of the two foods was used only for 2 days, after which the cat food was used for the rest of the rearing period. The same amount of the larval diet was provided to both the infected and uninfected groups. Three biological replicates were performed for each group. Pupal development time in the trays was monitored and recorded. Pupae were collected at 8-hr intervals and transferred to $13-\mathrm{mm}$ culture tubes. Later, emerging adults were sexed into males and females, and their emergence time was recorded.

\section{Wing size measurement}

Wing sizes were used to estimate adult body sizes as described previously with a slight modification [23]. Adult mosquitoes that were nearly 7- 8 days old, post-eclosion, were killed with ethyl acetate. The wings were carefully broken with forceps at the apex of the alular region, dipped into the alcohol to remove folding, and mounted on a slide. The slides were then photographed under a microscope at $4 \times$ magnification. Linear measurement, from the humeral cross vein to the wing tip, excluding fringe, was conducted using Axio vision software (Carl Zeiss). Intact wings of adults from both the infected and uninfected groups were measured for size variation analysis.

\section{Mating competitiveness assays}

Four adult cages were prepared with equal numbers of uninfected males and females (as outlined in Additional file 1: Table S1). Into these cages, varying numbers of infected males $(0,35,100$, or 200$)$ were released so that the ratio of uninfected females: uninfected males: infected males was either 1:1:0, 1:1:1, 1:1:2 or 1:1:4. Mosquitoes were allowed to mate for 2 days. The mosquitoes were then blood-fed for approximately $20 \mathrm{~min}$. Two days after blood-feeding, eggs cups were inserted into the cages for harvesting eggs. Eggs were collected for two nights. Egg hatching was then determined as described above. A second blood meal was given to the mosquitoes one week after the first feeding, and new collections of eggs were then made. The data from the two egg collections were pooled, and the egg hatch was measured and compared to an expected hatch rate, assuming equal competition between LB1 and LIS males [24].

\section{Life span assays}

In the first experiment, the life span was measured when mosquitoes were maintained on sucrose alone. Twentyfive mosquitoes (1-2 days old), either males or females, were transferred to small cages designed from plastic bowls in which a $10 \%$ sucrose solution was available. In the second experiment, the female life span was measured after the mosquitoes were fed a blood meal. Females (4-5 days old) were fed on commercial human blood through a membrane feeding apparatus. The next day, the unfed females were removed, and $\sim 40$ bloodfed females were transferred to $20 \times 20 \times 20$-cm cages. The mosquitoes were again allowed to take a blood meal; feeding was repeated at least four additional times, with a 6- to 7-day interval between each blood meal. For both experiments, dead mosquitoes were removed every day and recorded until no viable mosquitoes were left. Data from three replicate experiments were used for the survival assay.

\section{Statistical analysis}

The results of experiments other than the mating competitive and survival assays were first checked for normality of distribution using the D'agostino and Pearson omnibus normality test. The results of this test were used to decide whether to use a parametric or a nonparametric test for further analysis. To compare the mating performances of the infected and uninfected males, chi-squared goodness-of-fit was used to analyze expected and observed hatchings in cages with varying ratios of males. Kaplan-Meier survival analysis (log-rank tests) was used for adult survivorship. GraphPad Prism version 5.00 for Windows was used for data analyses.

\section{Results}

wAlbB reduces fecundity in LB1 mosquitoes

In order to examine the impact of $w$ AlbB on the fecundity of An. stephensi, we measured the number of eggs laid per LB1 female and the proportion of those eggs that hatched into larvae, as compared to wild-type LIS and the aposymbiotic line LBT mosquitoes. When 
mosquitoes were fed on mouse blood, there was no significant difference in the number of eggs laid by each female between the LB1 and LIS mosquitoes or between the LIS and LBT mosquitoes, although the LBT females laid slightly fewer eggs than did the LB1 females $(\mathrm{P}<0.05$, Student's $t$-test) (Figure 1A). The egg hatch rate of the LB1 mosquitoes (50.0\%) was significantly lower than that of either the LIS (73.0\%; $\left.\mathrm{P}<0.0001, \mathrm{X}^{2}=998.1\right)$ or LBT (74.6\%; $\mathrm{P}<0.0001, \chi^{2}=686.3$ ) mosquitoes (Figure 1B). Similar results were observed when the mosquitoes were fed on sheep blood: LB1 and LIS mosquitoes laid a similar number of eggs, but the egg hatch rate of the LB1 mosquitoes $(50.2 \%)$ was lower than that of the LIS mosquitoes (65.7\%; $\mathrm{P}<0.0001, \mathrm{X}^{2}=42.7$ ) (Figure $\left.1 \mathrm{C}, \mathrm{D}\right)$. We also compared the egg hatch rate of the LB1 and LBT mosquitoes after they had fed on human blood. Consistently, the number of eggs laid by the LB1 females was higher than that of the LBT mosquitoes $(\mathrm{P}<0.0001)$ (Figure $1 \mathrm{E})$, but the hatching rates of the LB1 mosquitoes (46.19\%) were still significantly lower than those of the LBT strain (61.97\%; $\mathrm{P}<0.0001, \mathrm{X}^{2}=119.7$ ) (Figure 1F).

\section{wAlbB has no impact on the life history traits and sex ratio of the LB1 mosquitoes}

In order to determine whether $w$ AlbB influenced mosquito larvae survivorship and development, we measured 1) the survivorship from L1 larvae to pupae and adults, 2) the development time from L1 larvae to pupae and from pupae to adults, and 3) the adult sex ratio. As shown in Table 1 , there was no significant difference between LB1 and LIS mosquitoes in survivorship during development from the L1 larval stage to pupae/adults. Both LB1 and LIS took a similar time to develop from L1 larvae to pupae and from pupae to adults. Furthermore,
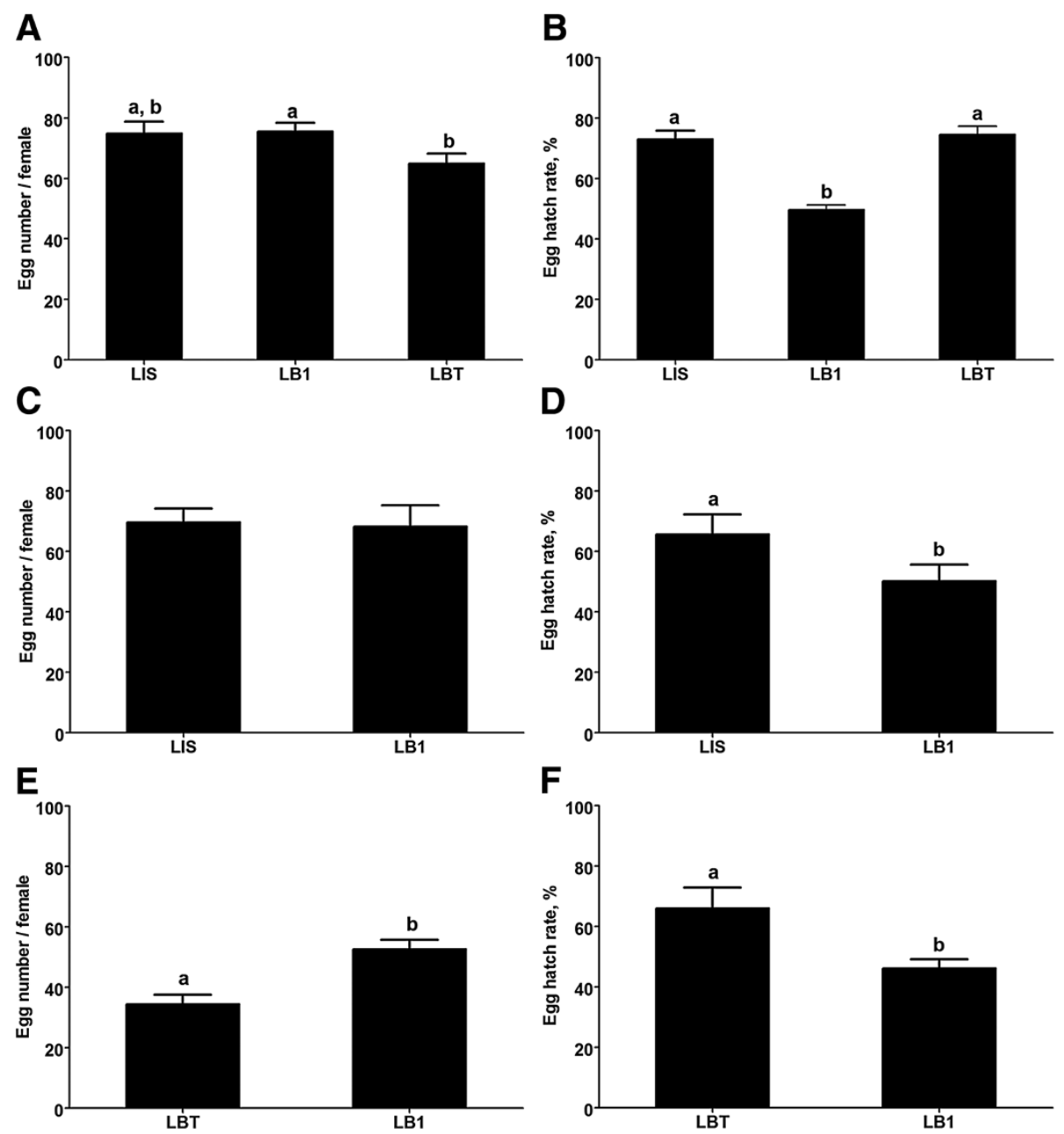

Figure 1 Impact of wAlbB on An. stephensi fecundity. The number of eggs laid by each individual female and the hatch rate after feeding on mouse (A, B), sheep (C, D) or human (E, F) blood. For all figures, error bars represent standard error; statistical significance is represented by letters above each column, with different letters signifying distinct statistical groups $[\mathrm{P}<0.05$; Student's $t$-test for $(\mathbf{A}$ and $\mathbf{E})$; $\mathrm{P}<0.0001$; chi-squared test for (B, D and F)]. 
Table 1 Life history attributes of wAlbB Wolbachia-infected and uninfected An. stephensi

\begin{tabular}{lccc}
\hline Attributes & LIS & LB1 & P-value \\
\hline Survivorship from L1 to pupa, \% & $89.00 \pm 3.21$ & $89.33 \pm 4.04$ & 1 \\
Pupation time, hr & $205.05 \pm 3.03$ & $194.75 \pm 11.78$ & 0.2164 \\
Survivorship from L1 to adult, \% & $80.67 \pm 5.86$ & $82.67 \pm 4.16$ & 0.655 \\
Female ratio, \% & $55.34 \pm 3.25$ & $50.56 \pm 11.64$ & 0.7229 \\
Male emergence time, hr & $238.04 \pm 5.79$ & $227.68 \pm 8.9$ & 0.1485 \\
Female emergence time, hr & $244.68 \pm 0.76$ & $238.07 \pm 7.56$ & 0.2075
\end{tabular}

An assumption of a 1:1 sex ratio was made when larvae were placed into rearing pans. Immature data are based on three replicates. For pupation time, pupa emerging within 5 consecutive days were used for analysis. During this time, pupal emergence in each rearing pan was $>85 \%$. Very few of the larvae which were still at $3^{\text {rd }}$ or $4^{\text {th }}$ instar at this time, were considered slow-growing and excluded from analysis. Fisher's exact test was used for comparison of survivorship and sex ratio, and Student's $t$-test was used for comparisons of development time.

no significant difference was observed in the sex ratios of LB1 and LIS mosquitoes.

wAlbB has no impact on the body size of LB1 mosquitoes Insect body size reflects changes in the environment and can be an indicator of overall fitness. To determine whether $w$ AlbB has any impact on the body size of LB1 mosquitoes, we measured the wing size of both females and males at 7-8 days old, after eclosion. We saw no significant difference in the wing size of the LIS, LB1, and LBT mosquitoes in both sexes (Figure 2A, B).

\section{wAlbB has a minor impact on LB1 males' mating competition}

The ability of LB1 males to compete with LIS males for mating with LIS females is important for developing a population suppression strategy. Therefore, we investigated mating competition by using laboratory population cages. Four cages containing different ratios of LIS females to LIS males to LB1 males (1:1:0, 1:1:1, 1:1:2 and 1:1:4) were set up. When only LIS males were present, the egg hatch rate reached $85.3 \%$ (Figure 3; Additional file 1: Table S1). The expected egg hatch (Figure 3; Additional file 1: Table S1) was calculated assuming equal competitiveness of LB1 and LIS males [24] and based on an egg hatch rate of $1.2 \%$ in the CI matings [7]. A comparison between the observed egg hatch and the expected egg hatch (above) showed that there was no significant difference between the two groups at the ratios of 1:1:2 and 1:1:4. Only a minor (1.78\%) deviation was observed at $1: 1: 1$, but this difference is statistically significant $\left(P<0.001, X^{2}=11.7\right)$ (Figure 3; Additional file 1: Table S1).

\section{wAlbB increases longevity of sugar-fed LB1 mosquitoes but not blood-fed LB1 females}

Previous studies have shown that $w \mathrm{AlbB}$ may provide a fitness advantage to mosquito hosts by increasing their
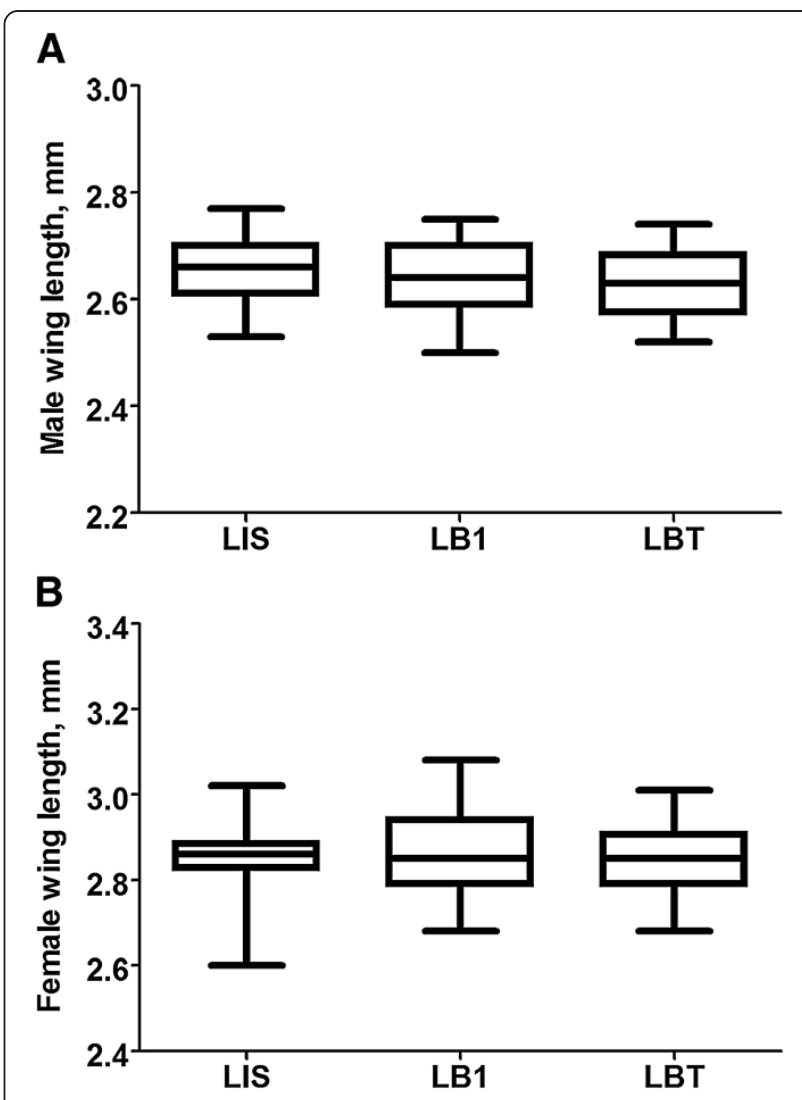

Figure 2 Impact of wAlbB on the body size of An. stephensi. Box plots display the observed distribution of wing length in female (A) and male (B) LIS, LB1, and LBT mosquitoes. The boxes in each panel represent (from bottom to top) the 25th to 75 th percentiles. Horizontal bars within the boxes indicate the median value of each group. Interactions between wAlbB Wolbachia infection and body size, as based on measurement of the wing size, were not detected in either sex $[P=0.7746$ for $(\mathbf{A}) ; P=0.487$ for (B); one-way ANOVA].

longevity. To determine whether a similar impact would be produced in An. stephensi, we first compared the longevity of females (not blood-fed) and males between LB1 and LIS mosquitoes when they were maintained on $10 \%$ sucrose alone. We found that both sexes of LB1 mosquitoes lived significantly longer than did LIS mosquitoes (log-rank test, $\mathrm{p}<0.01$ ) (Figure 4A, B). Although both LB1 and LIS males had a median longevity of 16 days, the median LB1 female longevity (22 days) was 6 days longer than that of LIS females (16 days).

We then compared the longevity of LB1 and LIS females after feeding on human blood. There was no significant difference between the two groups (log-rank test, $P>0.05)$. Both LB1 and LIS females had a median longevity of 15 days. LB1 females appeared to survive better during the first 10 days, whereas LIS females showed better survivorship later (after Day 26) (Figure 4C). 


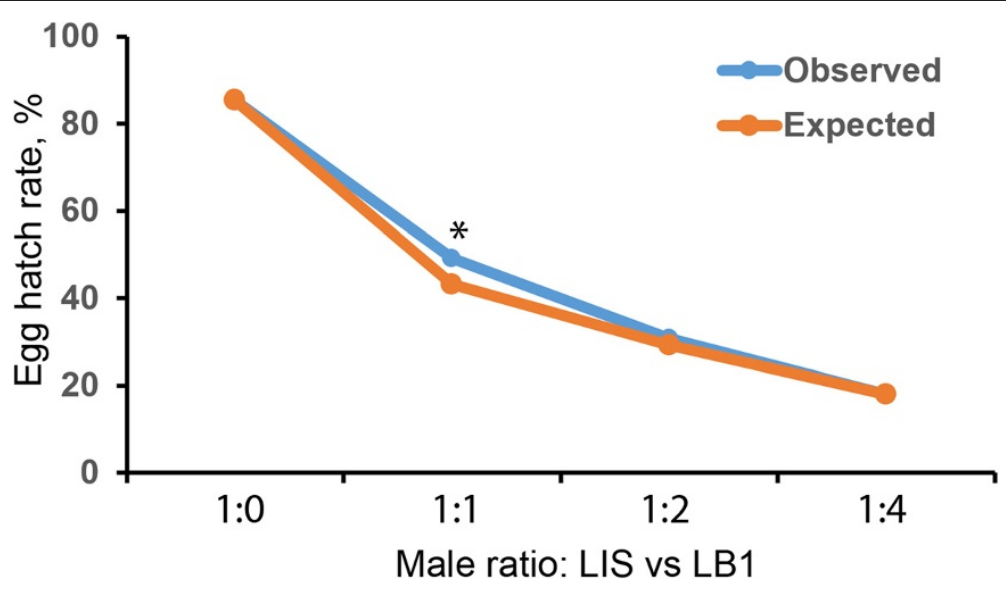

Figure 3 Impact of $w A l b B$ on male mating competitiveness. Suppression of egg hatch in LIS populations via release of LB1 males. The blue line illustrates the egg hatch observed in population cage tests. The orange line illustrates the expected egg hatch, assuming equal competitiveness of LB1 and LIS males [24].

\section{Discussion}

We have previously shown that the Wolbachia strain $w$ AlbB forms a stable association with An. stephensi, invades laboratory populations of this mosquito, and induces resistance to $P$. falciparum in this mosquito vector [7]. To facilitate the development of Wolbachiabased strategies for malaria vector control, we examined $w$ AlbB-associated fitness in An. stephensi in the present study. We found that Wolbachia wAlbB did not influence the egg numbers laid by LB1 females, but it reduced the egg hatch rate. Neither survivorship and development from L1 larvae to adults nor the adult sex ratio was influenced by $w$ AlbB. $w$ AlbB did have a very minor impact on the mating competitiveness of LB1 males. Longevity comparison experiments showed that both female and male wAlbB-infected An. stephensi lived significantly longer than their wild-type counterparts when the mosquitoes were fed on sugar meals alone. However, there was no difference in longevity between the LB1 and LIS females when they were given blood meals.

A reduction in egg hatch rate was consistently observed in LB1 mosquitoes fed mouse, sheep, or human blood. This result differed from that observed for Ae. albopictus, the original host of wAlbB, in which Wolbachia confers a reproduction advantage [16]. No fitness cost was seen when $w$ AlbB was stably introduced into Ae. aegypti either [5], suggesting that this fitness-related effect is hostspecific and is not only determined by the Wolbachia strain. A reduction in egg hatch rate was also observed in Ae. aegypti carrying a stable wMelPop infection, but mainly when the mosquitoes fed on nonhuman blood [25]; when they fed on human blood, however, only a mild decrease was observed [25]. We examined the hatch rate of LB1 eggs produced by females fed on human blood through an artificial feeding approach and found that the reduced egg hatch could not be restored, indicating that a different mechanism is responsible for the low egg hatch.

The reduction in LB1 egg hatch rate may be the result of an alteration in mosquito physiology, including changes in amino acids and ROS levels as a result of Wolbachia infection. A recent study has shown that the egg viability defect in Wolbachia-infected Ae. aegypti can be partially rescued by dietary supplementation with amino acids, supporting the possibility that the low egg hatch may be caused by a manipulation of amino acids [26]. For example, Wolbachia may compete with the mosquito host for amino acids, resulting in a lack of sufficient nutrition for embryonic mosquito development. Previous studies have also shown that a strain of $A n$. gambiae that was genetically selected to be refractory to malaria parasites has significantly higher levels (2 to 3 times) of hemolymph $\mathrm{H}_{2} \mathrm{O}_{2}$ than do unselected strains [27]. However, this enhanced immunity to Plasmodium infection came with an adverse effect on fecundity, which could be restored by supplementation of mosquitoes with antioxidants [27]. Similarly, the ROS level in the fat body, midgut, and whole body of LB1 mosquitoes are significantly higher than LIS mosquitoes [7], and this increase is likely to be one of mechanisms that mediates refractoriness to malaria parasites. Because ROS detoxification by catalase is a major determinant of fecundity in the mosquito An. gambiae, the elevated ROS in LB1 mosquitoes may not be able to be cleared by catalase, resulting in a reduction in egg hatch. Future studies should determine whether supplementation of LB1 mosquitoes with amino acids and antioxidants can restore this decline in fecundity.

The impact of Wolbachia on the immature stage has been reported in various hosts [28,29], and a recent model has predicted that even those Wolbachia infections that 


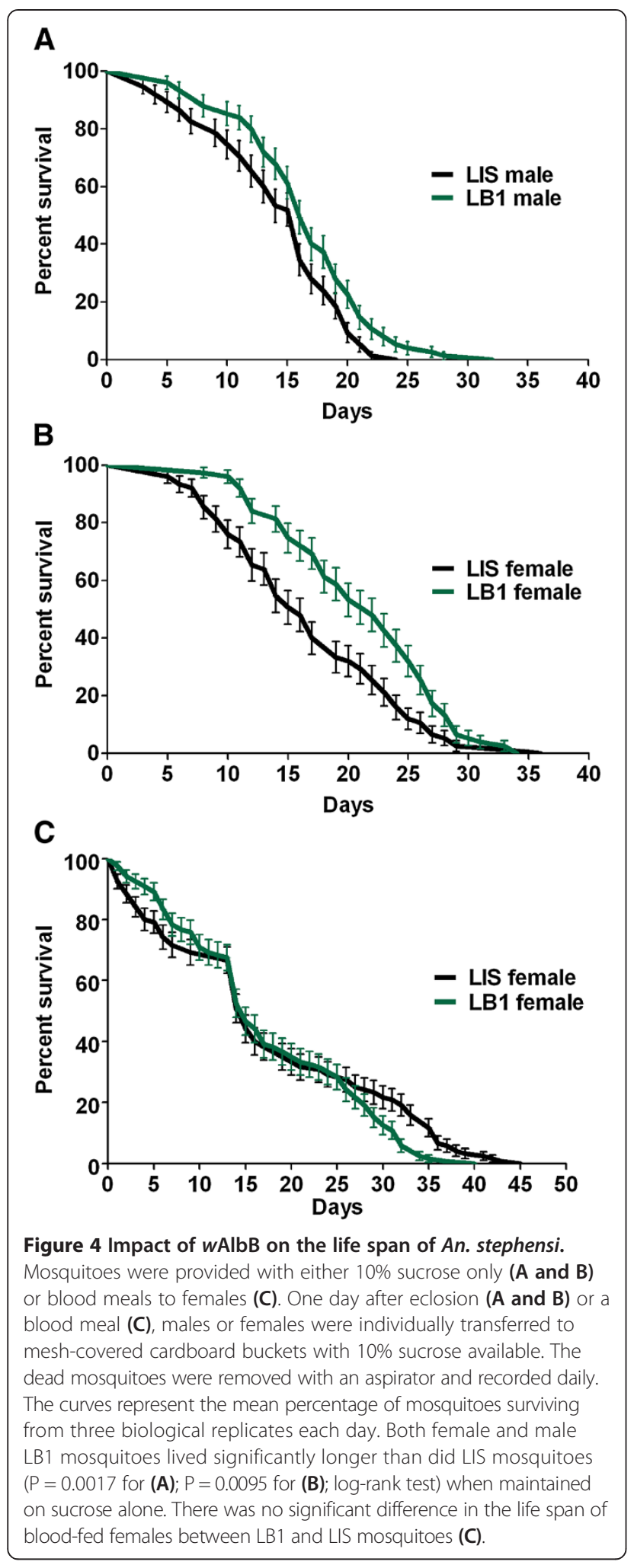

cause minor decreases in immature survival are unlikely to invade and spread within the host population [30]. In Aedes mosquitoes, a negative impact of Wolbachia infections on larval survival and development time has been observed $[28,29]$. Here, we did not see any effect of $w$ AlbB on survivorship during the development from L1 larvae to pupae/adults in LB1 versus LIS mosquitoes, consistent with the ability of $w$ AlbB to invade laboratory An. stephensi population cages. However, our experiments were conducted at a relative low larval density. Previous studies have shown that Wolbachia-infected mosquito larvae experience reduced survival when intraspecific competition is intense [31,32]. It would be of interest to know whether a similar result would be obtained with LB1 mosquitoes.

Body size is an important indicator of a number of mosquito fitness traits [33,34]. With higher energy reserves, larger females may have a better flight range, higher survival, increased host-finding and blood-feeding success, and improved ability to locate oviposition sites, while larger males may live longer and have more mating success [35]. In addition, mosquitoes with a larger body size may take a larger blood meal, resulting in an increase in their intake of Plasmodium gametocytes and development of high infection intensity [36]. However, we saw no significant difference in the wing size in the LIS, LB1, and LBT mosquitoes. This result is similar to that of a previous report in Ae. aegypti that the differences in the body size can largely be attributed to nutrition and, to a minor extent, to wMel Wolbachia infection [35].

Previous studies have indicated that mating competitiveness may be negatively affected in the transinfected male mosquitoes. After transfer of $w$ Ri Wolbachia into Ae. albopictus, a reduction in mating competitiveness was observed in comparison to wild-type mosquitoes [37]. However, no significant difference was found between the competitiveness of the wildtype and transinfected males after $w \mathrm{Mel}$ was transferred to Ae. albopictus [17]. Thus, the impact on competitiveness may be related to the Wolbachia strain used. We observed a very minor negative effect of $w$ AlbB on mating competitiveness between LB1 and wild-type males only at the ratio of 1:1:1. When more LB1 males were released, with the ratio increased to $1: 1: 2$ and 1:1:4, the observed egg hatch rate was not significantly different from the expected values, assuming an equal competitiveness of LB1 and LIS males, and suggesting that there is no significant difference in mating capacity between LB1 and LIS males. Together with the ability of LB1 males to induce nearly complete CI when mating with wild-type females, this strong mating competitiveness supports the prediction that mass release of LB1 males could lead to a potential local eradication of An. stephensi. This population suppression strategy has been successful in controlling Culex and Aedes mosquitoes in the field $[21,22]$.

We found that wAlbB Wolbachia increased the longevity of An. stephensi in both sexes when mosquitoes were maintained on sucrose alone; however, there was 
no difference in the longevity of infected and uninfected females after they took a blood meal. As the original native host of $w \mathrm{AlbB}$, Ae. albopictus lives significantly longer than the aposymbiotic line [16]. When $w$ AlbB is transferred into and forms a stable association with $A e$. aegypti, an increase in the longevity of the transinfected line is also observed [10]. An impact of Wolbachia on host longevity has also been found in other strains. Transfer of wMel into Ae. albopictus leads to an increase in longevity only in the male, whereas $w$ MelPop decreases the longevity of both native Drosophila and transinfected Aedes [17-19]. Thus, Wolbachia-host interactions must influence a mechanism that can determine host longevity. For example, the insulin/IGF-I signaling pathway is an evolutionarily conserved mechanism of longevity that is present from yeast to humans [38]. In Drosophila, Wolbachia has been reported to increase the fly's insulin/IGF-like signaling [39]. It would be interesting to know whether Wolbachia changes the host's life span by modulating the insulin/IGF-I signaling pathway.

The longevity of the mosquito vector contributes significantly to its vector capacity for malaria transmission. Because malaria parasites need to complete their development through an extrinsic incubation period in mosquitoes before being transmitted to humans, the older mosquitoes are more dangerous from the human perspective because they play more important roles in disease transmission. Although we observed an increase in the life span of LB1 females when they were maintained on sucrose alone, the LB1 females did not live significantly longer than the LIS females after taking human blood. In particular, LB1 females showed a trend toward living a shorter time than LIS females after Day 26. Additional experiments should be conducted to determine whether there is a change in the longevity of LB1 females after feeding on parasite-infected blood. It also should be noted that Wolbachia may clear all the parasites through its pathogen interference mechanism and leave all the older mosquitoes free of parasites. This possibility is supported by our recent observation that the Wolbachia density increases when LB1 mosquitoes age (data not shown).

On the other hand, the fitness advantage seen in the life span of LB1 mosquitoes that had not been blood-fed could have a positive impact on control strategies. For example, the LB1 females may survive better than LIS females do in an environment in which blood resources are limited or temporarily unavailable. This effect may counteract the negative impact of their low egg hatch, reduce the initial release threshold, and accelerate population replacement, resulting in improved efficacy when it is deployed as a practical malaria intervention strategy. Furthermore, only males will be released in the planned population suppression strategy. The increased longevity in the transinfected males may improve their mating capacity, resulting in more $\mathrm{CI}$ matings and stronger suppressive effects.

\section{Conclusions}

The ability to stably introduce $w$ AlbB into An. stephensi makes it possible to develop Wolbachia-based strategies for malaria vector control. Similar strategies have been successfully tested in field settings, and progress is being made toward eliminating dengue and lymphatic filariasis in a number of disease-endemic countries [6,22]. Both this prior experience and mathematical models suggest that the host fitness associated with Wolbachia is one of the key factors that determines the population dynamics following its field release. Our results have shown that $w$ AlbB induces both a fitness cost and benefits in $A n$. stephensi under laboratory conditions. All the data we have now accumulated for LB1 males, including their strong mating competitiveness, increased life span when taking sugar meals, and ability to induce nearly complete CI, support the feasibility of a population suppression/ eradication strategy. While LB1-mediated population replacement has been demonstrated in the laboratory condition [7], the LB1 fitness data reported here could facilitate the development of an improved mathematic modeling guiding the next field trial in the malaria endemic areas where An. stephensi is a primary vector. However, the laboratory measures used here, while informative, may not exactly reflect the realities of a competitive field environment. There may be difference in the fitness associated with Wolbachia infection between the long-established laboratory colonies and the field mosquitoes. Future studies should focus on whether Wolbachia-associated fitness is subject to changes in environmental or field conditions, such as temperature, humidity, and blood source; how these changes can influence the outcome of malaria control; and how all this information can be utilized to develop better control strategies.

\section{Additional file}

Additional file 1: Table S1. Mating competitiveness between wAlbB-infected and uninfected An. stephensi males.

\section{Competing interests}

The authors declare that they have no competing interests.

Authors' contributions

DJ and ZX designed research; DJ, MJM, DB and FZ conducted experiments; DJ and ZX. analyzed data and wrote the paper. All authors read and approved the final version of the manuscript

\section{Acknowledgments}

This work was supported by the National Institutes of Health/National Institute for Allergy and Infectious Disease Grant R01A1080597, a grant from the Foundation for the NIH through the Grand Challenges in Global Health 
Initiative of the Bill and Melinda Gates Foundation, and the Key Project of Chinese Ministry of Education (No. 311030). We are grateful to Deborah McClellan for editing this manuscript.

Received: 11 April 2014 Accepted: 13 July 2014

Published: 21 July 2014

\section{References}

1. Hilgenboecker $K$, Hammerstein $P$, Schlattmann $P$, Telschow A, Werren JH: How many species are infected with Wolbachia?-A statistical analysis of current data. FEMS Microbiol Lett 2008, 281(2):215-220.

2. Werren JH, Baldo L, Clark ME: Wolbachia: master manipulators of invertebrate biology. Nat Rev Microbiol 2008, 6(10):741-751.

3. Kittayapong P, Baisley KJ, Baimai V, O'Neill SL: Distribution and diversity of Wolbachia infections in Southeast Asian mosquitoes (Diptera: Culicidae). J Med Entomol 2000, 37(3):340-345.

4. Sinkins SP: Wolbachia and cytoplasmic incompatibility in mosquitoes. Insect Biochem Mol Biol 2004, 34(7):723-729.

5. Xi Z, Khoo CC, Dobson SL: Wolbachia establishment and invasion in an Aedes aegypti laboratory population. Science 2005, 310(5746):326-328.

6. Hoffmann AA, Montgomery BL, Popovici J, Iturbe-Ormaetxe I, Johnson PH, Muzzi F, Greenfield M, Durkan M, Leong YS, Dong Y, Cook H, Axford J, Callahan AG, Kenny N, Omodei C, McGraw EA, Ryan PA, Ritchie SA, Turelli M, O'Neill SL: Successful establishment of Wolbachia in Aedes populations to suppress dengue transmission. Nature 2011, 476(7361):454-457

7. Bian G, Joshi D, Dong Y, Lu P, Zhou G, Pan X, Xu Y, Dimopoulos G, Xi Z: Wolbachia invades Anopheles stephensi populations and induces refractoriness to Plasmodium infection. Science 2013, 340(6133):748-751.

8. Dobson SL, Bourtzis K, Braig HR, Jones BF, Zhou W, Rousset F, O'Neill SL: Wolbachia infections are distributed throughout insect somatic and germ line tissues. Insect Biochem Mol Biol 1999, 29(2):153-160.

9. Moreira LA, Iturbe-Ormaetxe I, Jeffery JA, Lu G, Pyke AT, Hedges LM, Rocha BC, Hall-Mendelin S, Day A, Riegler M, Hugo LE, Johnson KN, Kay BH, McGraw EA, van den Hurk AF, Ryan PA, O'Neill SL: A Wolbachia symbiont in Aedes aegypti limits infection with dengue, Chikungunya, and Plasmodium. Cell 2009, 139(7):1268-1278.

10. Bian $G, X u Y, L u P, X i e ~ Y, X i Z$ : The endosymbiotic bacterium Wolbachia induces resistance to dengue virus in Aedes aegypti. PLoS Pathog 2010 6(4):e1000833.

11. Kambris Z, Cook PE, Phuc HK, Sinkins SP: Immune activation by lifeshortening Wolbachia and reduced filarial competence in mosquitoes. Science 2009, 326(5949):134-136.

12. Teixeira L, Ferreira A, Ashburner M: The bacterial symbiont Wolbachia induces resistance to RNA viral infections in Drosophila melanogaster. PLOS Biol 2008, 6(12):e2.

13. Glaser RL, Meola MA: The native Wolbachia endosymbionts of Drosophila melanogaster and Culex quinquefasciatus increase host resistance to West Nile virus infection. PLoS One 2010, 5(8):e11977.

14. Walker T, Johnson PH, Moreira LA, Iturbe-Ormaetxe I, Frentiu FD, McMeniman CJ, Leong YS, Dong Y, Axford J, Kriesner P, Lloyd AL, Ritchie SA, O'Neill SL, Hoffmann AA: The wMel Wolbachia strain blocks dengue and invades caged Aedes aegypti populations. Nature 2011, 476(7361):450-453.

15. Hoffmann AA, Turelli M, Harshman LG: Factors affecting the distribution of cytoplasmic incompatibility in Drosophila simulans. Genetics 1990, 126(4):933-948.

16. Dobson SL, Marsland EJ, Rattanadechakul W: Mutualistic Wolbachia infection in Aedes albopictus: accelerating cytoplasmic drive. Genetics 2002, 160(3):1087-1094.

17. Blagrove MS, Arias-Goeta C, Di Genua C, Failloux AB, Sinkins SP: A Wolbachia wMel transinfection in Aedes albopictus is not detrimental to host fitness and inhibits Chikungunya virus. PLoS Negl Trop Dis 2013, 7(3):e2152.

18. McMeniman CJ, Lane RV, Cass BN, Fong AW, Sidhu M, Wang YF, O'Neill SL: Stable introduction of a life-shortening Wolbachia infection into the mosquito Aedes aegypti. Science 2009, 323(5910):141-144.

19. Min KT, Benzer S: Wolbachia, normally a symbiont of Drosophila, can be virulent, causing degeneration and early death. Proc Natl Acad Sci U S A 1997, 94(20):10792-10796.

20. Atyame CM, Pasteur N, Dumas E, Tortosa P, Tantely ML, Pocquet N, Licciardi S, Bheecarry A, Zumbo B, Weill M, Duron O: Cytoplasmic incompatibility as a means of controlling Culex pipiens quinquefasciatus mosquito in the islands of the south-western Indian Ocean. PLoS Negl Trop Dis 2011, 5(12):e1440.

21. Laven H: Eradication of Culex pipiens fatigans through cytoplasmic incompatibility. Nature 1967, 216:383-384.

22. Brelsfoard CL, Sechan Y, Dobson SL: Interspecific hybridization yields strategy for South Pacific filariasis vector elimination. PLoS Negl Trop Dis 2008, 2(1):e129.

23. Styer LM, Meola MA, Kramer LD: West Nile virus infection decreases fecundity of Culex tarsalis females. J Med Entomol 2007, 44(6):1074-1085.

24. Arunachalam N, Curtis CF: Integration of radiation with cytoplasmic incompatibility for genetic control in the Culex pipiens complex (Diptera: Culicidae). J Med Entomol 1985, 22(6):648-653

25. McMeniman CJ, Hughes GL, O'Neill SL: A Wolbachia symbiont in Aedes aegypti disrupts mosquito egg development to a greater extent when mosquitoes feed on nonhuman versus human blood. J Med Entomol 2011, 48(1):76-84.

26. Caragata EP, Rances E, O'Neill SL, McGraw EA: Competition for Amino Acids Between Wolbachia and the Mosquito Host, Aedes aegypti. Microb Ecol 2013, 67(1):205-218.

27. DeJong RJ, Miller LM, Molina-Cruz A, Gupta L, Kumar S, Barillas-Mury C: Reactive oxygen species detoxification by catalase is a major determinant of fecundity in the mosquito Anopheles gambiae. Proc Natl Acad Sci U S A 2007, 104(7):2121-2126.

28. McMeniman CJ, O'Neill SL: A virulent Wolbachia infection decreases the viability of the dengue vector Aedes aegypti during periods of embryonic quiescence. PLoS Negl Trop Dis 2010, 4(7):e748.

29. Islam MS, Dobson SL: Wolbachia effects on Aedes albopictus (Diptera: Culicidae) immature survivorship and development. J Med Entomol 2006 43(4):689-695

30. Crain PR, Mains JW, Suh E, Huang Y, Crowley PH, Dobson SL: Wolbachia infections that reduce immature insect survival: predicted impacts on population replacement. BMC Evol Biol 2011, 11:290.

31. Gavotte L, Mercer DR, Stoeckle JJ, Dobson SL: Costs and benefits of Wolbachia infection in immature Aedes albopictus depend upon sex and competition level. J Invertebr Pathol 2010, 105(3):341-346.

32. Gavotte L, Mercer DR, Vandyke R, Mains JW, Dobson SL: Wolbachia infection and resource competition effects on immature Aedes albopictus (Diptera: Culicidae). J Med Entomol 2009, 46(3):451-459.

33. Xue RD, Barnard DR, Muller GC: Effects of body size and nutritional regimen on survival in adult Aedes albopictus (Diptera: Culicidae). J Med Entomol 2010, 47(5):778-782

34. Ponlawat A, Harrington LC: Factors associated with male mating success of the dengue vector mosquito, Aedes aegypti. Am J Trop Med Hyg 2009, 80(3):395-400

35. Yeap HL, Endersby NM, Johnson PH, Ritchie SA, Hoffmann AA: Body size and wing shape measurements as quality indicators of Aedes aegypti mosquitoes destined for field release. Am J Trop Med Hyg 2013, 89(1):78-92.

36. Vezilier J, Nicot A, Gandon S, Rivero A: Insecticide resistance and malaria transmission: infection rate and oocyst burden in Culex pipiens mosquitoes infected with Plasmodium relictum. Malar J 2010, 9:379.

37. Xi Z, Khoo CC, Dobson SL: Interspecific transfer of Wolbachia into the mosquito disease vector Aedes albopictus. Proc Biol Sci 2006, 273(1592):1317-1322.

38. Barbieri M, Bonafe M, Franceschi C, Paolisso G: Insulin/IGF-I-signaling pathway: an evolutionarily conserved mechanism of longevity from yeast to humans. Am J Physiol Endocrinol Metab 2003, 285(5):E1064-E1071.

39. Ikeya T, Broughton S, Alic N, Grandison R, Partridge L: The endosymbiont Wolbachia increases insulin/IGF-like signalling in Drosophila. Proc Bio/ Sci 2009, 276(1674):3799-3807.

doi:10.1186/1756-3305-7-336

Cite this article as: Joshi et al:: Wolbachia strain wAlbB confers both fitness costs and benefit on Anopheles stephensi. Parasites \& Vectors 2014 7:336. 